\title{
Los sentidos construidos por jóvenes de Caleta Olivia sobre el derecho a la educación: un estudio sobre los efectos de la racionalidad estatal ${ }^{1}$
}

\section{The meaning built by young people of Caleta Olivia on the right to education: a study on the effects of state rationality}

Eduardo Langer, Mariela Cestare, Huayra Martincic

langereduardo@gmail.com ${ }^{2}$, mcestare@ hotmail.com ${ }^{3}$, huayramartincic@gmail.com ${ }^{4}$ Universidad Nacional de la Patagonia Austral. Unidad Académica Caleta Olivia.

Recibido: 12/03/2019. Aceptado: 05/07/2019

\section{RESUMEN}

El presente trabajo indaga en los sentidos construidos por los y las jóvenes de poblaciones vulnerables sobre problemáticas vinculadas a las condiciones en que se ejerce el derecho a la educación en el marco de las sociedades del gerenciamiento. El objetivo es rastrear la pregunta acerca de los efectos de la racionalidad estatal en las construcciones que los sujetos hacen en relación con su escolaridad. La hipótesis central que desarrollaremos es que al quedar los sujetos librados a la gestión de sí, en Caleta Olivia se viene produciendo, desde hace algunos años, una situación de precariedad en torno al acceso, la permanencia y el tipo de educación en el nivel secundario. Frente a dicha situación, se profundizan las desigualdades en las escolaridades de los y las adolescentes y jóvenes. Sin embargo, a pesar de estas realidades inciertas y precarias, ellos y ellas aún apuestan por sostener sus formaciones. Para ello, este trabajo se realiza a partir de abordajes metodológicos que incluyeron instancias cualitativas -mediante técnicas de investigación tales como la observación participante y entrevistas en profundidad - así como cuantitativas mediante la realización de encuestas a 412 estudiantes que asisten a escuelas secundarias públicas de la localidad. Algunos de los resultados que se mostrarán se vinculan con las posibilidades construyen sobre el derecho a la educación atendiendo a la importancia que otorgan a la escuela, las formas en que esta prepara para ser un ciudadano/a responsable y para participar en organizaciones estudiantiles.

Palabras clave: Derecho a la educación; Jóvenes; Sentidos; Racionalidad estatal; Poblaciones vulnerables.

\footnotetext{
${ }^{1}$ El presente trabajo se realizó a partir de una Beca UNPA de Iniciación a la Investigación, el marco del PI "Luchas por los derechos sociales y construcción de saberes en comunidades en condición de pobreza." Dir. Eduardo Langer. El mismo se encuentra incluido en las actividades del área socio-pedagógica.

2 Doctor en Educación, Prof. Adjunto Sociología de la Educación. CONICET/UNSAM/UNPA. langereduardo@gmail.com

3 Prof. en Ciencias de la Educación. Asistente de docencia en la cátedra Pedagogía. UNPA. mcestare@ hotmail.com

${ }^{4}$ Becaria UNPA. ICIC. UACO.
} 


\begin{abstract}
The present investigational work inquiries on vulnerable populations young people built up senses about problematics vinculated to the conditions in which the educational right is applicated in the management societies framework. The goal is to trace the query on the estate rationality effects on the constructions the subjects make on the relation to their scholarship. The central hypothesis we have developed studies when the subject is left to the management of itself. In Caleta Olivia since a few years now is occurring this situation of precariousness around the access, the permanency and the kind of education in the high school level. Before the situation already mentioned, it deepens the teenagers and young adults inequalities Nerveless, besides this uncertain and precarious realities they still lay down on their academic formation. That for this job is carried on based on methodological approaches that have included qualitative instances -through investigation techniques such as the observation of the partaker and in-depth interviews-, as well as qualitative by performing quizzes on 412 students which attend to local public high schools on the daily basis some of the results to show are vinculated to the possibilities this schools young people build and grant about the educational right attending the purport around the school, about the ways in which it's prepared to become a responsible citizen and to participate on student's organizations.
\end{abstract}

Key words: Education rights; Young people; Meaning; State rationality; Vulnerable populations.

\title{
INTRODUCCIÓN
}

El presente trabajo indaga en los sentidos construidos por los y las jóvenes de poblaciones vulnerables sobre problemáticas vinculadas a las condiciones en que se ejerce el derecho a la educación en el marco de las sociedades del gerenciamiento (Grinberg, 2008). Estas sociedades expresan centralmente racionalidades en las que los sujetos se encuentran ubicados en el centro de la escena porque son los únicos y principales responsables de sus éxitos o fracasos en función de sus propias capacidades para gestionar los medios que disponen para lograr aquello que se proponen. Por ello, el objetivo es rastrear como pregunta los efectos de esas racionalidades estatales en las construcciones que los sujetos hacen en relación con su escolaridad. La hipótesis central que desarrollaremos es que al quedar los sujetos librados a la gestión de sí, en Caleta Olivia se viene produciendo, desde hace algunos años, una situación de precariedad en torno al acceso, la permanencia y el tipo de educación en el nivel secundario. Frente a dicha situación, se profundizan las desigualdades en las escolaridades de los y las adolescentes y jóvenes ya que al asumir que cada uno debe volverse responsable de sí mismo, se desconocen y se ubican en segundo plano las condiciones de existencia en la que cada sujeto tiene que vivir.

En el caso de Caleta Olivia, no puede obviarse que se trata de una localidad en que en los últimos años, la ciudad ha atravesado una serie de complicaciones en la vida cotidiana. En este sentido, y para dar cuenta de lo señalado, se recuperan algunas de las problemáticas que han estado - y están actualmente - en la agenda pública, y con presencia en los medios de comunicación. Entre los más destacados pueden nombrarse: la falta de suministro de agua de manera continua; el clima constante de malestar social; las condiciones insalubres a raíz de la falta de recolección, tratamiento de residuos y los desbordes cloacales; el mal funcionamiento del transporte público. De igual modo, sectores tan variados como el de salud, educación, administración provincial y municipal y trabajadores de actividades de la construcción y del 
sector petrolero - muy importante en la vida económica de la región -, cooperativistas y beneficiarios de planes sociales y ayuda financieras, llevan adelante reclamos por mejoras salariales y de condiciones de trabajo. El paro, la retención de tareas y el corte de ruta son ya acciones cotidianas de la localidad, presentes en el cotidiano de hace ya algunos largos años.

La realidad educativa de Caleta Olivia se ve atravesada, a su vez, por problemáticas propias. A nivel provincial, a las suspensiones por la emergencia hídrica, se suman el levantamiento de clases ya que las condiciones edilicias no permiten el normal desarrollo de las actividades en las instituciones (falta de gas, de luz, de mobiliario e incluso de espacios aptos para funcionar como aulas); también la falta de docentes - o el hecho de que hay horas cátedras sin cubrir -. Por otra parte, las medidas de fuerza del sector han generado que en 2017 hubiese 105 días de paro; en 2016 noventa y cinco días sin clases y en 2015 más de cuarenta; situación irresuelta desde años anteriores. Ante este panorama, cada vez son más los casos que - siempre y cuando tengan los medios para hacerlo - derivan por parte de la familia en la búsqueda de vacantes en colegios de gestión privada. La situación educativa se encuentra en un conflicto profundo, que pareciera no tener una solución próxima, a tal punto, que se han hecho públicos casos de estudiantes y docentes que deciden migrar a otras provincias, a vivir con familiares para poder acceder a la educación formal. En este marco, la racionalidad estatal en la localidad de Caleta Olivia tiene efectos sobre esos sentidos que los y las jóvenes van construyendo en relación al derecho a la educación y ante este escenario, signado por la vulneración de derechos básicos, ellos buscan el modo de garantizar el cumplimiento efectivo de su escolaridad, desarrollando estrategias de diversa índole para sostenerla frente a una sociedad que se presenta en constante crisis (Langer, Cestare, Villagran, 2015) y pese a los contextos desfavorables en que se encuentran. Las acciones que los y las adolescentes y jóvenes - y sus familias - desarrollan para lograr sostener su escolaridad dan cuenta del valor que le asignan a la escuela y la educación en general.

Por tanto, el presente trabajo de investigación se propuso generar conocimiento sobre los sentidos que los y las jóvenes construyen en torno a la educación, atendiendo a la importancia que le otorgan a la escuela, sobre las formas en que prepara para ser un ciudadano/a responsable y para participar en organizaciones estudiantiles. También, profundizamos sobre los sin sentidos e incertidumbres que se van produciendo en y desde el sistema educativo estatal y por las cuales los y las estudiantes van adoptando diferentes miradas y posicionamientos en relación a buscar que se garantice ese derecho. Enmarcados en el campo de la sociología de la educación, así como desde los estudios de gubernamentalidad y en relación a estudios sobre juventudes en y desde el sur, se realiza aquí la descripción de los sentidos que los y las jóvenes escolarizadas construyen en torno al derecho a la educación y a las contradicciones e incertidumbres que se generan para poder garantizar ese ejercicio.

Se espera aportar una mirada de la comunidad en términos afirmativos, constructivos, de posibilidad y potencia para desnaturalizar y criticar discursos deterministas y fatalistas sobre jóvenes en contexto de pobreza. Para ello se realizaron entrevistas en profundidad, registros de observación en escuelas secundarias y el relevamiento de encuestas, en el marco de un PDTS, en el que se relevó y analizó resultados en torno a los sentidos que construyen los jóvenes sobre la importancia de la escuela y las formas en que prepara para el ejercicio de la ciudadanía y para participar en organizaciones estudiantiles.

El trabajo está estructurado a partir de dos primeros apartados de breve enmarcamiento histórico, legal y teórico del problema en cuestión. Posteriormente, se analizarán algunos resultados del trabajo de campo sobre los sentidos de los y las jóvenes y las tensiones que 
ubican entre los procesos de inclusión y de exclusión que las racionalidades van produciendo así como las contradicciones y las pugnas que van mostrando para sostener la escolaridad.

En el marco del trabajo se procuró un acercamiento a los escenarios, las relaciones y las variables en torno al acceso, permanencia y tipo de escolaridad a través de una investigación de carácter exploratoria y cualitativa. Siguiendo a lo expuesto por Taylor y Bogdan (1987), estas entrevistas se caracterizaron por ser flexibles, dinámicas y no estructuradas. Fueron más conversaciones entre iguales donde asumimos un rol que no sólo buscó la respuesta a preguntas preestablecidas, sino que fuimos aprendiendo qué preguntas hacer y cómo hacerlas. Fueron reiterados encuentros cara a cara "dirigidos hacia la comprensión de perspectivas que tienen los informantes respecto de sus vidas, experiencias o situaciones, tal como las expresan con sus propias palabras" (Taylor y Bogdan, 1987: 101).

Por otro lado, en el marco del PDTS $^{5}$ en el que también esta beca se desarrolló, se implementaron 412 encuestas a estudiantes en escuelas secundarias públicas de la localidad durante el año 2018, cuyos datos son tomados y, algunos de ellos, analizados aquí. Estás encuestas tuvieron la finalidad de indagar acerca de la miradas de los y las jóvenes sobre la escuela y algunas de las dimensiones trabajadas fueron el uso de la tecnología, las ideas sobre la escuela y el barrio, la formación, el futuro, las regulaciones sobre las conductas y la formación para el trabajo. Como adelantamos, a los fines de este informe, recuperamos y analizamos los resultados en torno a los sentidos que construyen sobre la escuela y su importancia según NBI, en segundo lugar, los sentidos sobre la forma que prepara la escuela para ser un ciudadano/a responsable según NBI y, por último, los sentidos sobre las formas en que prepara la escuela secundaria para participar en organizaciones estudiantiles según niveles de NBI.

\section{MARCO DE REFERENCIA}

\subsection{El derecho a la educación entre la responsabilidad estatal y la individual.}

En Argentina, el derecho a la educación - comprendido como el derecho de enseñar y aprender, se encuentra ya presente en la constitución de 1853. En ese momento, el mismo se encontraba contenido dentro de las libertades individuales de cada ciudadano. De manera paulatina, esta primera caracterización fue dando paso a la idea de que el derecho a la educación no sólo refería a un derecho de cada persona, sino que debía ser comprendido como un derecho social, dado su alcance e importancia para el conjunto de la sociedad. Esta visión se oponía a la idea de la educación como derecho individual, y a la educación como mercancía.

Comprender la educación como derecho social significaba que debía ser protegido y asegurado por el Estado, de modo tal que todos pudieran acceder a la educación. Sin embargo, ello no implicaba que dicha educación fuera igual para todos. De hecho, al mismo tiempo que se asumía la importancia de que el Estado se encargara de la educación de la población en el caso de Argentina, fuertemente vinculado a la formación del Estado Nación y la necesidad de homogeneizar a la población-, persistía la idea de que la educación era un privilegio para las élites (Puiggrós, 2003, Pineau 2001), por lo cual, si bien los amplios sectores populares eran

\footnotetext{
${ }^{5}$ PDTS 538 "Centro de Intercambio y Reservorio de Información Social y Educativo (CIRISE)" en la región del Golfo San Jorge. Primera Etapa: Educación Secundaria. Dirigido por Silvia Grinberg.
} 
incluidos, esta inclusión no se daba de manera absoluta ni en todos los niveles. Era un sistema que, siguiendo a Abratte (2015) priorizo el acceso a los niveles superiores de educación sólo a los sectores sociales privilegiados, a la vez que amplió el acceso de los sectores populares a la escuela elemental, más elemental aún para los más pobres, los pobladores rurales, los inmigrantes excluidos del circuito laboral, las mujeres, entre otros sectores sociales. Por decirlo de alguna forma, de manera progresiva y sostenida el derecho a la educación en Argentina se ha encontrado vinculado a las acciones que realiza el Estado para asegurar el acceso de toda la población a la escolaridad elemental, formal y obligatoria.

A su vez, el ejercicio de este derecho se encuentra atravesado por las tensiones respecto de las diferencias entre las clases populares y las élites. A través de los años, el derecho a la educación se ha mantenido como un derecho clave, vinculado a la formación y ejercicio de la ciudadanía y ha ido ampliando los alcances. Así, en el presente se vincula con el ejercicio del derecho a la educación no sólo el acceso a la escuela (Torres, 2006) sino que da cuenta del derecho de toda persona a acceder a una educación, que brinde posibilidades de acceso y participación en procesos de aprendizaje vinculados a los saberes considerados socialmente valiosos y significativos, que permitan a la vez reconstruir los procesos de activación de esos saberes -en momentos de crisis- para generar emprendimientos cooperativos promoviendo el devenir de los saberes del trabajo en saberes socialmente productivos (Puiggros y Gagliano 2004). Es decir, se produce una paulatina expansión de la universalización de la educación obligatoria, que permite a todos los habitantes del país acceder a la escuela. Mientras la Ley de Educación Común (Ley $\mathrm{N}^{\circ}$ 1420) gratuita y obligatoria, promulgada en 1884, establecía la obligatoriedad de la educación primaria, su sucesora - la Ley Federal de Educación $\left(\mathrm{N}^{\circ}\right.$ 24.195) ampliaba dicha obligatoriedad, modificando la distribución de la educación general básica e incluyendo dos años más, así como hacia obligatorio un año del nivel inicial. Con la Ley de Educación Nacional No 26.206/06, la educación obligatoria incluye el Nivel Inicial y la totalidad de la Educación Secundaria.

Asimismo, desde hace algunos años, es centro de debate el derecho a la educación superior. Aunque no puede negarse que esta expansión ha permitido ampliar la cantidad de sujetos que acceden a la escolaridad, dicho proceso puede caracterizarse como una expansión condicionada (Gentili, 2009), proceso mediante el cual los sistemas nacionales de educación tendieron a crecer en una dinámica de segmentación y diferenciación institucional que otorga a los individuos que transitan sus circuitos, estatus y oportunidades altamente desiguales. De este modo, si bien son cada vez más los años de escolaridad obligatoria - y por tanto, que el Estado debe garantizar-, los y las jóvenes no reciben las mismas oportunidades educativas. El proceso de expansión de la matricula se ha dado de manera paralela a una continua diferenciación, segmentación y fragmentación de la educación (Braslavsky 1989, Llomovate 1993, Tiramonti 2009, Bowles y Gintis 1981, Baudelot y Establet 1976).

Estos debates en torno al acceso desigual al derecho a la educación se encuentran presente en las reflexiones en torno a quienes están dentro y fuera del sistema. Hay una serie de trabajos que indagan respecto del derecho a la educación (Abratte; 2015; Torres; 2006); el modo en que se vincula la desigualdad social y económica con la desigualdad educativa (Gentili; 2009; Scabuzzo y Fabrizi, 2014); y las posibilidades de acceso y ejercicio del derecho a la educación en los sectores vulnerables (Langer y Cestare, 2015; Langer, Perez, Cestare; 2015; Langer y Cestare; 2014; Langer, 2012; Langer, Roldán y Maza; 2012; Grinberg, 2009; Pérez, 2013, Cestare, 2007; Venturini, 2007; Villagrán, 2016, Langer, 2011). Estos trabajos dan cuenta de las dificultades que se presentan para que el acceso a la escolaridad encuentre correlación en la permanencia y egreso de la educación secundaria, especialmente en aquellas 
poblaciones que se encuentran en condiciones de vulnerabilidad. Estas poblaciones viven, tal como señala Butler (2017), situaciones de vida provocadas y reproducidas por las instituciones gubernamentales y económicas sobre la base del trabajo temporal, en donde se suprimen los servicios sociales y se produce la erosión generalizada de cualquier vestigio de democracia social, imponiendo en su lugar modalidades empresariales que se apoyan en una feroz defensa ideológica de la responsabilidad individual.

Tal como dijimos con anterioridad, hay expansión de la escolaridad pero también hay un derecho que sigue siendo negado (Gentili, 2009) en una sociedad de gran polarización y desigualdad social que ha profundizado la brecha entre quienes poseen recursos y quienes no tienen acceso a ellos promoviendo situaciones en que los sujetos se enfrentan diariamente a una realidad que los ubica cada vez más ante situaciones de mayor vulnerabilidad. Esos efectos de racionalidades que hacen responsables a los individuos y a las instituciones ante todo, van calando en los sentidos, los intereses y las "elecciones" totalmente condicionadas de los sujetos y sobre los accesos al conocimiento y al aprendizaje.

Teniendo en cuenta el hecho de que el derecho a la educación se encuentra presente en el artículo 26 de la Declaración Universal de los Derechos Humanos, es importante atender a que el derecho a la educación no sólo refiere a la escolarización obligatoria. Atender al derecho a la educación es sostener que todas las personas tienen derecho a la educación y que el objeto de la educación será siempre el pleno desarrollo de la personalidad humana y el fortalecimiento del respeto a los derechos humanos y a las libertades fundamentales. Resulta pertinente preguntarse si, pese a los avances en la legislación que promueven el acceso a la educación, nos enfrentamos a una realidad en la que no hay un ejercicio real de los derechos pese a que los sujetos traccionan, pugnan y demandan por más y mejores derechos (Langer, 2017). Son esos sentidos los que expresan los caminos a recorrer, las demandas de las poblaciones y los desafíos por lograr el acceso, pero también estar, aprender y vivir la cotidianeidad de las instituciones.

\subsection{Sentidos en contextos de incertidumbres}

Se considera a los sentidos como no individuales ni personales. Tal como dice Deleuze (1969), son las emisiones de las singularidades que son los verdaderos acontecimientos trascendentales y que presiden la génesis de los individuos y de las personas. Así, siguiendo al autor, el sentido es lo que expresa la proposición como acontecimiento que insiste o subsiste. No existe fuera de la proposición que lo expresa, es una atribución, que aún sin ser atributo de la proposición, lo es de la cosa o del estado de las cosas. Estos sentidos expresan las creencias personales y generales sobre la escuela, la formación en tiempos de exclusión y de desocupación.

A pesar de las sociedades en crisis, de los contextos de pobreza urbana y de los procesos de fragmentación educativa es posible proponer que desde los diferentes sentidos que expresan los y las estudiantes, la escuela continúa siendo un espacio de integración, de estructuración de proyectos y expectativas de vida e incide para poder pensar proyectos de vida. Los discursos de la caída de valores y la decadencia que, generalmente, son atribuidos a los y las jóvenes, según Saintout (2009), son una expresión clara del miedo que produce este nuevo tiempo. A pesar de la incertidumbre, de la desorientación que, muchas veces, parece imperar en la escuela, las contradicciones políticas y normativas, las idas y vueltas, los temores, miedos, angustias y pérdidas, nos encontramos con estudiantes que hablan de deseos, sueños, cambio, amor, odios. Hablan de ellos, de sus familias, de la inseguridad y la angustia que 
produce encontrarse reiteradamente con caminos sin salidas o con las miradas negativas de los otros. Relatan sus dificultades para darle significado a lo que se enseña en la escuela y cómo se enseña (Langer, 2014).

Tal como dijimos, esos sentidos entran en relación con las racionalidades que se ponen en juego, con las condiciones históricas que hacen posible el desarrollo de una determinada política educativa, dinámicas y prácticas escolares, relaciones entre saber y poder que desatan ciertas estrategias y técnicas gubernamentales en las instituciones como la escuela y, tal como lo dice Perea Acevedo (2009), implica hacer visible lo invisible, analizar y evidenciar las racionalidades en las que estos dispositivos emergen, se mantienen y se transforman. Así, la racionalidad es inmanente a los micropoderes, es una forma de ejercicio del gobierno y del poder que apunta al conjunto de prácticas a través de las cuales se pueden constituir, definir, organizar e instrumentalizar las estrategias que los individuos pueden establecer unos en relación a otros -o sea individuos libres que intentan controlar, determinar, delimitar la libertad de los otros-, y para hacerlo disponen de ciertos instrumentos para gobernarlos (Foucault, 1994). Es decir, se gobierna a través de la conducción de la conducta, la racionalidad política y la tecnología de gobierno (De Marinis, 1999).

Los programas políticos, las estrategias y las tecnologías están orientados a hacer algo en relación a un objetivo practicable (O’Malley, 1996), por ello las racionalidades políticas no simplemente surgen como resultado de la lógica de poder sino que son desarrolladas con propósitos específicos. Se trata de situar la discusión en torno de los sentidos de los y las estudiantes en contextos de pobreza urbana, desde los estudios de la gubernamentalidad, recuperando las diferentes formas de racionalidad, de instrumentalizar la conducta y las esferas de prácticas destinadas de diversos modos a controlar los individuos y las colectividades (Lemke, 2006). Interesa destacar, especialmente en relación al objeto de estudio, cómo se apela a las capacidades autorregulatorias de los individuos y de las comunidades para producir la economización más efectiva de los medios de gobierno. Las formas actuales que presenta el gobierno de la población donde los individuos, las comunidades o los grupos devienen responsables, supone a la vez desplazar las responsabilidades por riesgos sociales como la enfermedad, la desocupación, la pobreza, la educación y la responsabilidad misma de vivir en sociedad situándola en el dominio de la cuestión individual o comunitaria, transformándola así en un problema de gestión de sí (Rose, 2007; Grinberg, 2008).

En este estudio, nos referimos a jóvenes escolarizados. Como etapa de la vida, media entre la madurez biológica y la madurez social, es un periodo de permisividad, donde las demandas sociales - a la incorporación al ámbito productivo, formar un hogar - son menores y se cuenta con un tiempo "preparatorio". Esto implica que el ser "joven" no se condice de manera directa con la categoría estadística, o al menos, implica que existe más de una juventud. Esto se profundiza aún más desde hace algunas décadas, dado que ya no existe un contexto de estructuras sociales que habiliten la construcción de identidades y normas que permitan delimitar con cierta claridad seguridades y obligaciones. En lugar de las redes de contención que antes garantizaban sus derechos, hoy los sujetos deben resolver por sí mismos las situaciones que se les presentan, dentro del marco de incertidumbre que implica el capitalismo flexible. Por tanto, los sentidos de los y las jóvenes están asociados a los efectos de las racionalidades estatales en este marco histórico. Es decir, en sociedades en crisis, de precariedad y de incertidumbres. Sus sentidos se van produciendo en esta trama y formación histórica. 
Esta política de la incertidumbre nos agita, nos disloca, nos empuja, nos sujeta, trastoca nuestra vida (Sennett, 2000) y, por si fuera poco, nos hace sentir culpables de nuestro "fracaso". Ante esta política, nos culpabilizamos por las consecuencias en nuestra vida. En este contexto, la estigmatización de los y las jóvenes se vuelve dato central y permanente de la problemática para entender el proceso de desplazamiento de la conflictividad social hacia los mismos individuos. Según Castel (2004), es el retorno de las clases peligrosas, situadas en los márgenes, como toda representación de la amenaza que entraña en sí una sociedad. La "población liminar" (Foucault, 2006) suele vivir en una cantidad de situaciones caracterizadas por la precariedad, por la incertidumbre social a gran escala y el incremento de la fricción de lo incompleto (Appadurai, 2007), creando una ansiedad intolerable con los bienes provistos por el Estado, desde la vivienda, la educación, la salud hasta la seguridad. Es por ello que allí, donde una o varias de estas formas de incertidumbre social entran en juego, la violencia puede crear una macabra forma de certeza y puede convertirse en una técnica brutal acerca de "ellos" y, por lo tanto, acerca de "nosotros" (Appadurai, 2007). Es decir, la incertidumbre junto con lo incompleto, producen ira, descontento, aunque, también, temor y falta de tolerancia.

A la vez, la incertidumbre hace que las personas vivan al mismo tiempo en lo legal y lo ilegal, que no se distinga entre lo privado y lo público, que se reciba un salario y la ayuda social, que se pase del empleo al desempleo y viceversa de un día a otro, que se viva del día y de la noche, que se pida al Estado y a la sociedad (Mallimaci y Salvia, 2005). A los sujetos que viven en esas condiciones de pobreza se los denomina como los "condenados", "abandonados", "perdidos" porque están todos los días al borde de la muerte y viven o se baten contra ella, son los "rechazados", las "deyecciones", los "síntomas de la patología del cuerpo social" listos para abandonar la incertidumbre de su círculo y pasar al de la condena (Onfray, 2011). Pero también, es en las fisuras que supone la actual economía política de la incertidumbre que "se levantan acciones que en la lucha cotidiana por sobrevivir constituyen prácticas de resistencia y lucha" (Grinberg, 2006). En el marco de estas luchas, nos referiremos a los sentidos que los y las jóvenes expresan sobre el derecho a la educación atendiendo a la inclusión, a su importancia y al ejercicio de la ciudadanía así como de la participación estudiantil.

\section{RESULTADOS Y DISCUSIÓN: sentidos sobre la inclusión, la importancia de la escuela y el ejercicio de la participación}

A fin de describir los sentidos que construyen los y las jóvenes sobre el derecho a la educación, se presentan algunos primeros resultados de una encuesta, realizada por el equipo del área socioeducativa (UNPA-UACO), en el marco de un PDTS en que se inscribe el proyecto que enmarca esta beca. Así mismo, se recuperan algunos breves registros de observación ilustrativos de clases de una escuela de Caleta Olivia. En el primer caso, la información recabada permite reconstruir los sentidos (Deleuze; 1994) construidos en torno a la escuela secundaria por parte de los y las estudiantes que asisten diariamente a ellas. Mientras que en el segundo, se atiende a los discursos de los adolescentes y jóvenes, en orden a reconstruir el valor que la escuela asume para ellos. Sostenemos que, aún frente a las contradicciones, sin sentidos e incertidumbres que surgen en el día a día social y escolar, los y las estudiantes confían en la escuela como un espacio que les ofrece un escenario en que es posible el ejercicio de los derechos, o en el que pueden buscar la efectivización de los mismos, tal como puede observarse en el siguiente registro: 
A partir de la presentación del tema de la clase, la docente pregunta a los estudiantes los motivos que pueden llevar a la realización de determinadas acciones. Los estudiantes se niegan a responder. La docente les pregunta por la actitud de no participar y ellos explican que en la hora anterior discutieron con la profesora por la realización de una actividad, en la que se les pidió su opinión y luego se les impuso lo que tendrían que hacer, por lo cual muchos sintieron que su palabra no fue tomada en consideración. La docente retoma la discusión para preguntar las razones por las cuales, pese a la situación planteada por los estudiantes, concurren y permanecen en la escuela, señalando que existen diferentes motivos para que los estudiantes estén allí, en clases; dice que existe la obligación de estar en la escuela, pero rápidamente señala que seguramente hay otros motivos más importantes. Una de las estudiantes dice que la escuela sirve para no morirse de hambre. Otra, que está ahí para aprender. Otro dice que a él no le queda otra. Finalmente, otro estudiante, que siempre está con los auriculares puestos y casi nunca participa, dice que él no tiene motivos, que él no quiere estar allí. La docente insiste "Pero estás acá", luego de un breve silencio, el estudiante dice que está ahi por el diploma". (Registro de observación, $4^{\circ}$ año, Agosto de 2018).

La variedad de respuestas de los y las estudiantes implica esa diversidad de situaciones en sus condiciones de vida, así como de las vivencias escolares que cada estudiante ha atravesado históricamente. Este registro de observación expresa cómo en un lapso menor a una hora de clase, se presentan dos docentes que interpelan de distinto modo a las y los estudiantes. Con diferentes formas y estilos, dan lugar a que surja un abanico de posibilidades en torno a qué hacer y qué esperar de la escuela. Para los y las estudiantes, la escuela se presenta como un espacio en el cual de manera simultánea, se constituye como lugar para la expresión de opiniones, al mismo tiempo que se imponen ciertos modos de ser, estar y pensar. Quizás, estas tensiones que se producen entre la inclusión y formas de exclusión escondidas, eufemizadas, cotidianas son aquellas que tienen más significado para pensar el derecho a la educación diario. Sin embargo, estas tensiones no son motivo suficiente para dejar de estar. La escuela sigue teniendo un valor central en sus vidas: tanto como espacio de aprendizaje, como de instancia que les permitirá "no morirse de hambre" o, incluso, para tener el diploma. Esas ideas de quedarse "porque no los queda otra" o "por el diploma" dan cuenta de que es la escuela un espacio en el que pueden estar, eligen estar. Entre estas tensiones, contradicciones e inmanencias es que nos ubicamos para describir los sentidos actuales, para caracterizar las formas en que los y las jóvenes se ubican y piensan la escuela hoy.

En este sentido, el trabajo realizado permite sostener que desde los sentidos que para ellos y ellas la escuela tiene como institución, es valorada no solo como espacio que permite acceder a saberes, sino también como lugar que permite ingresar en "mejores" condiciones al ámbito laboral o seguir estudiando. Es decir que la escuela se presenta desde muchos sentidos como institución que ofrece un panorama que aleja de la exclusión y de situaciones de marginación (Langer, Cestare, Martincic; 2018). Resulta necesario señalar que esto no significa que por estar incluidos en la escuela lo estén socialmente, y más en la sociedad en la que vivimos, que se caracteriza por no contar con espacios para todos. La desigualdad de las condiciones de vida de los y las jóvenes (Langer, Cestare y Villagran, 2015; Langer y Cestare, 2015) hace del derecho a la educación una discusión que cada vez es más difícil de afrontar. Aunque los y las estudiantes se encuentren "formalmente" incluidos, las condiciones de existencia en que se encuentran inmersos imposibilitan que esa inclusión sea real (Gentili, 2009) ya que esa escolarización está marcada por una dinámica de exclusión incluyente, vinculado a procesos apoyados en mecanismos de inclusión o inserción institucional que resultan insuficientes e 
inocuos para revertir aislamientos, marginación y negación de derechos involucrados en todo proceso de segregación social dentro y fuera de la escuela.

Sin desatender a estos aspectos, es interesante prestar atención al modo en que la escuela es significada como un espacio que permite pensar en otras posibilidades de vida. Especialmente, frente a un escenario en que los sujetos son responsables de sí en situaciones que resultan paradójicas, porque para que individuo pueda hacerse cargo de sí mismo bajo unas condiciones de precariedad generalizada, tal como plantea Butler (2017), se está dando por hecho algo asombroso, y es que se asume que las personas pueden (y deben) actuar de manera autónoma en unas condiciones en que la vida se ha hecho invivible. Las circunstancias en las que se estudia, tanto en lo que respecta a las condiciones de existencia de cada estudiante en su vida diaria, como aquellas vinculadas al funcionamiento de las escuelas, dan cuenta de las dificultades que se presentan para sostener ese espacio cuando se trata de escuelas situadas en comunidades en condición de pobreza.

De esta forma, cobra relevancia el par inclusión-exclusión tanto a nivel escolar como social, en la medida en que los actores educativos forman parte y reconocen esos procesos de exclusión aún estando incluidos en el sistema escolar y queriendo incluirse en el sistema social (Langer, 2018). Por ello pensamos en términos de dinámica, son jóvenes excluidos que pugnan por ser incluidos, son adultos que apuestan, sostienen y trabajan cotidianamente en esa inclusión, luchan cotidianamente contra la exclusión social que sufren sus estudiantes y ellos mismos. Son profesores que saben que son excluidos sus estudiantes y luchan para que puedan ser incluidos. Son autoridades que hacen el mismo movimiento. Quizás la pugna por la inclusión debe ser uno de los movimientos de resistencia más fuertes de los últimos tiempos/décadas en la educación (Langer, 2018). Siguiendo esta línea de pensamiento, observamos que son los y las jóvenes y sus familias son quienes deben asumir un papel activo para asegurar el cumplimiento del derecho a la educación, mediante una serie de estrategias que buscan sostener su presencia en las escuelas, quedando las comunidades libradas a la gestión de sí (Rose, 2007; Grinberg, 2008). En este sentido, observamos tensiones y contradicciones entre lo que efectivamente sucede en torno a la inclusión, acceso y permanencia, y como consecuencia de ello, cuando se indaga, se hace presente que los sentidos construidos en torno al derecho a la educación resultan contradictorios.

Como ya se ha señalado en otros trabajos, frente a relatos, particularmente desde los medios de comunicación (Langer, Cestare y Martincic; 2017) que refieren, en muchos casos a la apatía de los y las jóvenes, nos encontramos con relatos que dan cuenta de que estos jóvenes realizan acciones de resistencia frente a una realidad que se les presenta como dada e inmodificable. Es decir, que son jóvenes que apuestan por la escuela secundaria porque hallan en ella un valor que les permite proyectar un futuro mejor. La importancia de asistir a la escuela puede observarse desde las formas en que los y las jóvenes realizan acciones para sostener su escolaridad, aun cuando las condiciones son adversas, tal como se presenta en el siguiente registro:

Ingresa al aula una estudiante y dice que llegó tarde porque no le arrancaba la moto, que se le complicó. "Yo sabía que hoy teníamos sólo esta materia y vine igual, vine sólo por esta materia”. La docente dice que esperan un poco a que vengan más compañeros para comenzar y en esa espera establecemos una conversación en donde comenta que es mamá y que le cuesta a veces coordinar estudiar y cuidar de sus hijas (mellizas de dos años); señala que ella necesita conseguir trabajo, que estuvo trabajando, cuidando niños y haciendo limpieza de hogares, pero que este año se 
quedó sin trabajo, pero que en todo momento siguió yendo a la escuela. Que en algún momento pensó en dejar de estudiar para trabajar, pero que opto por seguir estudiando. (Registro de observación, julio de 2018, 4 año, 2018).

Seguir en la escuela aún en situaciones adversas, seguir pese a todo. Seguir porque la escuela ayuda y aporta, tensiona esa inclusión con sus propios procesos de exclusión, precariedad, incertidumbre. En estos contextos, es posible afirmar que los y las jóvenes construyen sentidos en torno a la escuela secundaria que se vuelven centrales para sus vidas en tanto los vinculan con el mundo del trabajo y los prepara para los estudios superiores y el ejercicio de la ciudadanía. De esta forma, los datos que expresa la Tabla $\mathrm{N}^{\circ} 1$ dan cuenta de la importancia de la escuela para los y las jóvenes en términos de la preparación para el trabajo, de los estudios posteriores, de la ciudadanía y del ser buena persona.

Tabla 1 - Sentidos de los y las jóvenes sobre la importancia de la escuela secundaria según niveles de NBI (Caleta Olivia - 2018). En \%. N=412

\begin{tabular}{|l|l|l|l|l|l|}
\hline & $\begin{array}{l}\text { NBI } \\
\text { Bajo }\end{array}$ & $\begin{array}{l}\text { NBI } \\
\text { Medio Bajo }\end{array}$ & $\begin{array}{l}\text { NBI } \\
\text { Medio }\end{array}$ & $\begin{array}{l}\text { NBI Medio } \\
\text { Alto }\end{array}$ & $\begin{array}{l}\text { NBI } \\
\text { Alto }\end{array}$ \\
\hline La escuela es importante para el trabajo & $83,1 \%$ & $86,3 \%$ & $81,8 \%$ & $65,9 \%$ & $84,1 \%$ \\
\hline $\begin{array}{l}\text { La escuela es importante para estudios } \\
\text { posteriores }\end{array}$ & $61,4 \%$ & $66,7 \%$ & $61,4 \%$ & $53,7 \%$ & $59,1 \%$ \\
\hline $\begin{array}{l}\text { La escuela es importante para ser un buen } \\
\text { ciudadano }\end{array}$ & $50,6 \%$ & $51,1 \%$ & $50,0 \%$ & $61,0 \%$ & $27,3 \%$ \\
\hline $\begin{array}{l}\text { La escuela es importante para ser buena } \\
\text { persona }\end{array}$ & $49,4 \%$ & $42,0 \%$ & $59,1 \%$ & $51,2 \%$ & $47,7 \%$ \\
\hline Es poco, pero la escuela algo hace por mí & $20,5 \%$ & $17,4 \%$ & $20,5 \%$ & $34,1 \%$ & $31,8 \%$ \\
\hline
\end{tabular}

Fuente: Elaboración propia en base a investigación en PDTS "Centro de Intercambio y Reservorio de Información Social y Educativo (CIRISE)”, con datos de NBI correspondiente al Censo Nacional INDEC 2010. Área Sociopedagogica - UNPA - UACO (2016-2018)

Aquí queremos focalizar la mirada en el ejercicio de los derechos, por tanto aquello que dicen para ser buen ciudadano/a. Los y las jóvenes dicen en un $50 \%$ o más que la escuela los prepara para ser buen ciudadano/a en todas las franjas de NBI de las escuelas exceptuando el caso de escuelas ubicadas en emplazamientos con NBI alto (27\% de los y las jóvenes encuestados afirman que la escuela los prepara para ser buenos ciudadanos). Es decir, en la escuela con mayores necesidades básicas insatisfechas nos encontramos con que un porcentaje considerablemente mayor de estudiantes afirma que la escuela no los prepara para la ciudadanía. Si consideramos la comparación con el resto de las respuestas para esos estudiantes, aún se hace más importante esta respuesta expresada. Los y las estudiantes de la escuela con mayor NBI piensan que la escuela es importante centralmente para el trabajo (84 $\%)$ y también para seguir estudios posteriores (59 \%). Pero no para el ejercicio de la ciudadanía. Por ello, al seguir indagando al respecto para profundizar estas diferenciaciones sobre la preparación para la ciudadanía entre estudiantes que asisten a escuelas con distintos NBI, observamos la Tabla $\mathrm{N}^{\mathrm{o}} 2$ : 
Tabla $N^{o} 2$ - Sentidos de los y las jóvenes sobre las formas en que prepara la escuela secundaria para ser un ciudadano/a responsable, según niveles de NBI (Caleta Olivia 2018). En \%. $N=412$.

\begin{tabular}{|l|l|l|l|l|l|l|}
\hline & NBI BAJO & $\begin{array}{l}\text { NBI Medio } \\
\text { bajo }\end{array}$ & NBI Medio & $\begin{array}{l}\text { NBI Medio } \\
\text { Alto }\end{array}$ & NBI Alto & Total \\
\hline Muy Bien & $44,6 \%$ & $32,6 \%$ & $34,1 \%$ & $30,8 \%$ & $38,0 \%$ & $35,7 \%$ \\
\hline Bien & $38,6 \%$ & $34,4 \%$ & $40,9 \%$ & $25,6 \%$ & $38,0 \%$ & $35,7 \%$ \\
\hline Regular & $8,4 \%$ & $24,9 \%$ & $13,6 \%$ & $10,3 \%$ & $6,0 \%$ & $16,0 \%$ \\
\hline Mal & $2,4 \%$ & $3,2 \%$ & $2,3 \%$ & $5,1 \%$ & $3,0 \%$ & $3,1 \%$ \\
\hline No responde & $6,0 \%$ & $5,0 \%$ & $9,1 \%$ & $28,2 \%$ & $15,0 \%$ & $9,4 \%$ \\
\hline & $100,0 \%$ & $100,0 \%$ & $100,0 \%$ & $100,0 \%$ & $100,0 \%$ & $100,0 \%$ \\
\hline
\end{tabular}

Fuente: Elaboración propia en base a investigación en PDTS "Centro de Intercambio y Reservorio de Información Social y Educativo (CIRISE)”, con datos de NBI correspondiente al Censo Nacional INDEC 2010. Área Sociopedagogica - UNPA - UACO (2016-2018)

En la Tabla $\mathrm{N}^{\mathrm{o}}$ 2, los y las jóvenes dan cuenta de una forma mucho más homogénea en que las distintas escuelas preparan para la ciudadanía responsable. Lo más importante que expresa esta tabla es que jóvenes que concurren a escuelas con NBI altos y bajos dicen que los prepara muy bien o bien en mayor medida para una ciudadanía responsable. No hay casi distinción entre las distintas escuelas. Es decir, los estudiantes rescatan, valoran y sostienen el ejercicio de derechos dentro del espacio escolar, sea la institución que sea. Aunque, también, hacen ciertas críticas a lo que no funciona bien como, por ejemplo, que no haya clases, que muchos docentes se ausenten, etc. Con este mismo sentido, ubicamos la discusión sobre las formas en que la escuela prepara o contribuye para la participación en organizaciones estudiantiles como parte de esos ejercicios de ciudadanía y de sostenimiento de los derechos de los estudiantes en el espacio escolar, tal como se trabaja en la Tabla $\mathrm{N}^{\circ} 3$.

Tabla $N^{o} 3$ - Sentidos de los y las jóvenes sobre las formas en que prepara la escuela secundaria para participar en organizaciones estudiantiles, según niveles de NBI (Caleta Olivia - 2018). En \%. $N=412$.

\begin{tabular}{|l|l|l|l|l|l|l|}
\hline & NBI BAJO & $\begin{array}{l}\text { NBI Medio } \\
\text { bajo }\end{array}$ & $\begin{array}{l}\text { NBI } \\
\text { Medio }\end{array}$ & $\begin{array}{l}\text { NBI Medio } \\
\text { Alto }\end{array}$ & NBI Alto & Total \\
\hline Muy Bien & $14,5 \%$ & $8,6 \%$ & $9,1 \%$ & $7,7 \%$ & $12,0 \%$ & $10,3 \%$ \\
\hline Bien & $18,1 \%$ & $22,2 \%$ & $36,4 \%$ & $30,8 \%$ & $26,0 \%$ & $24,2 \%$ \\
\hline Regular & $41,0 \%$ & $44,8 \%$ & $40,9 \%$ & $23,1 \%$ & $31,0 \%$ & $39,2 \%$ \\
\hline Mal & $10,8 \%$ & $19,0 \%$ & $4,5 \%$ & $5,1 \%$ & $8,0 \%$ & $12,9 \%$ \\
\hline No responde & $15,7 \%$ & $5,4 \%$ & $9,1 \%$ & $33,3 \%$ & $23,0 \%$ & $13,3 \%$ \\
\hline & $100,0 \%$ & $100,0 \%$ & $100,0 \%$ & $100,0 \%$ & $100,0 \%$ & $100,0 \%$ \\
\hline
\end{tabular}

Fuente: Elaboración propia en base a investigación en PDTS "Centro de Intercambio y Reservorio de Información Social y Educativo (CIRISE)", con datos de NBI correspondiente al Censo Nacional INDEC 2010. Área Sociopedagogica - UNPA - UACO (2016-2018)

En esta Tabla $\mathrm{N}^{\mathrm{o}}$ 3, observamos que para los jóvenes la escuela no contribuye, o lo hace muy poco, a la generación de espacios para la organización de los y las estudiantes. Si sumamos quienes no responden o que dicen que la escuela forma mal o regularmente para la participación en organizaciones estudiantiles, son aproximadamente el $65 \%$ de ellos que sostienen esos sentidos. Aquí, sí hay distinción por NBI de escuelas. Son en mayor medida los y las jóvenes estudiantes de escuelas "más acomodadas" geográficamente, que piensan de esta forma. Si observamos los sentidos en relación a que la escuela enseña bien o muy bien 
para la participación en organizaciones estudiantiles, si bien es menor el porcentaje total (34 $\%$ del total de los estudiantes), entre ellos hay una tendencia a que sean más aquellos estudiantes que están en escuelas con NBI medio alto o alto con respecto a los más bajos. Es decir, comprendiendo que el derecho a la educación no sólo debe dar cuenta de la posibilidad e instrucción si no de acceder a espacios que promuevan y favorezcan el desarrollo pleno de la persona, los y las estudiantes dan cuenta de dichos aspectos aunque con algunas diferencias: es reconocida como espacio que permite formarse para ser un buen ciudadano/a y buena persona, para seguir estudiando y para acceder al mercado laboral. Además, el derecho a la educación implica no sólo el derecho a acceder sin restricciones, pero también el derecho a aprender a avanzar y todo ello con rigurosidad y seriedad.

Entre las ideas del derecho a la educación y la educación de calidad, hay una relación de mutua determinación y de necesidad recíproca (Rinesi, 2016) que aquí no desarrollaremos ni profundizaremos. Solo mencionar que, tal como trabajamos con anterioridad en otros lugares (Langer, Pérez y Cestare, 2015), damos cuenta que los estudiantes esperan mucho más de la escuela de lo que se cree porque los y las docentes esperan en mayor proporción que los y las estudiantes que la escuela forme para ser buenos ciudadanos/as y buenas personas, en ambas áreas. En cambio, suceden tendencias diferentes en cuanto a la justificación de que sirve para continuar estudios posteriores. Así, se desmontan frases que se escuchan y producen en la escuela y en la sociedad sobre el no interés, ganas y gustos de los y las estudiantes. Cuando se empieza a indagar un poco más, ello no es lo que predomina. Frases que se expresan desde parte de la academia tales como "no hay mucho interés en la escuela" o el que la escuela "no sirve absolutamente para nada" (Kessler, 2002) no son expresiones que representen la sensación generalizada de la experiencia escolar por parte de los y las estudiantes en escuelas en contextos de pobreza urbana en Caleta Olivia. La asistencia a la escuela, aunque muchas veces discontinua, más que la pérdida de sentido de la escuela para los y las estudiantes en contextos de pobreza urbana, según Redondo (2004) evidencia una importante significación en las historias de los y las jóvenes, no necesariamente asociada a visiones románticas sobre su realidad ni a posiciones optimistas, sino más bien a una búsqueda de un espacio y un tiempo que los inscriba.

Sin duda, en esta tensión entre sociedades actuales que se caracterizan de manera cada vez más notoria por sujetos que deben volverse "artífices de su propio destino" y que son responsables de su suerte -aún cuando sus condiciones de existencia y, por tanto, las desigualdades existentes se van profundizando-, y las demandas y pugnas por más y mejor educación, es donde queremos instalar los efectos de las racionalidades estatales en los sentidos que los y las jóvenes van construyendo sobre el derecho a la educación. El "temor a la exclusión" es un proceso global (Bauman, 2014) y es desde allí que el mandato por la inclusión en la escuela secundaria, como receta que provienen de los organismos internacionales para los países en desarrollo como los latinoamericanos, se suma a la nave de miedos en tiempos de crisis. Ello quizás sea una de las principales y permanentes discusiones que se desarrollan en las escuelas, aún más cuando enfrentan períodos de reforma educativa.

Estas dinámicas se van internalizando y dejan huella en nuestra subjetividad, de modo que sin ser plenamente conscientes, esas "marcas" van condicionando el modo en que actuamos porque, siguiendo a Butler (2006), somos seres sociales hasta el más íntimo de los niveles seres que se comportan respecto de un "tú", fuera de sí mismos, constituidos por normas culturales que nos preceden y nos exceden, entregados a un conjunto de normas culturales y a un campo de poder que nos condiciona de manera fundamental (Butler, 2006). Quizás es por ello que los estudiantes van internalizando las regulaciones, las normas, las exclusiones. En 
esta biopolítica actual van internalizando los fracasos como propias responsabilidades, tal como en el siguiente registro de observación se expresa:

$N$. se considera "un mal alumno". Él lo expresó explícitamente en diferentes oportunidades. Al consultarle por qué decía eso, dijo "Yo no entiendo nada." y señaló que a él "Le iba mal". Le pregunté entonces si él eso se lo planteaba a los docentes, y me contestó que aprendió que debe mantenerse callado y "no molestar." Intenté entrevistarlo, pero no quería responder la entrevista, porque no quería decir cosas que lo hicieran quedar mal. (Registro de observación, $4^{\circ}$ año, Junio de 2018)

No entender nada, irle mal, mantenerse callado, no molestar, no quedar mal. Los estudiantes van internalizando los efectos de las racionalidades. Allí hay exclusión aunque esté adentro de la escuela. Hay una inclusión que no es plena. Hay un derecho que no se expresa en su totalidad. Es un joven que quiere estar en la escuela, a pesar de todo. Hay marcas y huellas profundas. También, sucede que esos sentidos dejan huella en la subjetividad, específicamente en las formas particulares que tienen los sujetos para enfrentar el entorno, en este caso la escuela, con sus dudas, incertidumbres y temores (Useche Aldana, 2008). Esos sentidos contradictorios, yuxtapuestos y tensionantes desarrollan nuevas formas de subjetividad (Rotker, 2000) y producen experiencias que actualizan constantemente las incertidumbres y afecta los cuerpos, tanto de estudiantes como así también de docentes que sufren somatizando cada vez con más frecuencia. En esos sentidos, hay miedos profundos que tienen el poder de afectar a los sujetos a través de las angustias individuales y colectivas, de las amenazas permanentes, la sensación de soledad y de desconfianza (Useche Aldana, 2008). Observamos que esos miedos de los y las jóvenes en la escuela ya no anticipan la inevitabilidad de los destinos, no conllevan fracaso, sino que son luchas contra esa inevitabilidad, luchas que se convierten en una tarea para toda la vida enfrentándose a sus raíces (Bauman, 2014) e implican reacciones y respuestas al desarraigo porque son luchas contra la injusticia y las diversas formas de exclusión (Barbero, 2000). Los sentidos que producen los y las estudiantes se realizan conjuntamente con las posibilidades de enfrentarlos, superarlos e insistir para poder cumplir los sueños y deseos propios. Se preguntan, tal como dice Rotker (2000), por las formas de cómo vencerlos, en términos del autor de cuerpo a cuerpo o de cuerpo con cuerpo. Por ello, no hay una renuncia total de los y las jóvenes, existe una demanda por otro tipo de prácticas y de experiencias, tal como se expresa en este último registro:

[Esto los profes tendrían que saberlo] "Que cuando expliquen, que no hagan gestos, porque yo a veces "No profe, no entendi" y me explicaban como dos, tres veces y tampoco lo entendía y ya ponían gesto y yo "Ah, sí profe, sí entendí." Y ya fue, para qué. O sea, si hacen esos gestos con la cara... ya me los conozco. Y después me llevan a Diciembre. Porque no todos entendemos. C. lo entiende de una, pero a mí me tienen que decir cinco o diez veces, para recién acordarme o entender qué cosa hay que hacer." (Entrevista a estudiante varón, 16 años. 25 de Junio de 2018).

Es decir, nos encontramos que esos efectos de las racionalidades estatales y las nuevas lógicas biopolíticas se tensionan con sujetos que ya no son meramente callados o sumisos ante ellas. Quieren estar en la escuela, quieren sostener la escolaridad, pelean por su derecho a la educación (así como tantos otros derechos). Quieren entender, quieren aprender, no quieren que los excluyan explícita o implícitamente. Estas tensiones y contradicciones son las que, quizás, nos encontramos en los sentidos que construyen cotidianamente los y las jóvenes frente a racionalidades que muchas veces expresan sin sentidos y desorganizaciones profundas. 


\section{CONCLUSIÓN}

A partir de la indagación sobre las características que asume el ejercicio del derecho a la educación de adolescentes y jóvenes enmarcado en las lógicas que asumen las sociedades del gerenciamiento (Grinberg; 2008), es que nuestro interrogante fue en torno a cuáles son los sentidos que construyen sobre ese derecho y las formas en que es condicionado por las racionalidades estatales.

De esta forma, describir los sentidos de los estudiantes desde su cotidiano permite concebir a la escuela como un espacio de interpretación e integración, de estructuración de proyectos y expectativas de vida. Allí pueden pensar y se piensan recurriendo a sentimientos tales como el amor, la soledad y la angustia que produce encontrarse reiteradamente con caminos sin salidas o con las miradas negativas de los otros. Hay tensiones profundas que se encuentran en esos sentidos, en los relatos de sus dificultades y de sus esperanzas dentro de la escuela, para poder sostener la escolaridad, para poder sostener el derecho a la educación. Tal como dice Grinberg (2008), "lo racional o, más bien, la racionalidad remiten al conjunto de principios que regulan y, por cierto, legitiman y guían la acción, a los criterios conforme con los cuales los sujetos controlan su entorno, a las máximas según las cuales se dirigen y actúan en el mundo. Por lo que la racionalidad remite a formas morales o éticas de administración de la vida” (p.95).

Desde la lectura de los datos nos preguntamos qué implica demandar y pugnar por derechos en contextos de precariedad, incertidumbre y exclusión en que parecieran que, cada vez más, es necesario reivindicarlos. Nuestro análisis comenzó con la hipótesis de que los y las estudiantes confían en la escuela como un espacio que les ofrece posibles, saberes, lugares, relaciones entre algunas de las cosas que rescatan prioritariamente. Como relatamos, para ellos y ellas la escuela les sirve para no morirse de hambre, para aprender, porque no queda otra, por el diploma, por las posibilidades de construir un proyecto mejor, para encontrarse con otros. Aún con todas las tensiones y contradicciones, decíamos que la escuela se presenta, desde muchos sentidos, como institución que ofrece algo distinto a la exclusión y las situaciones de marginación que viven cotidianamente. Estos sentidos que construyen están muy alejados de las visiones pesimistas y negativas de que a ellos no les importa nada, que no quieren estudiar y son unos vagos. Aunque no muy alejados de las racionalidades de hacerse cargo y gestionarse a sí mismos la vida, y en este caso, la escolaridad.

Como parte de los resultados centrales a los que arribamos en este trabajo, describimos las formas en que los jóvenes piensan que la escuela, sea la institución que sea, tanto escuelas con NBI altos y bajos, los prepara muy bien o bien para una ciudadanía responsable. Es decir, la totalidad de los y las estudiantes rescatan, valoran y sostienen el ejercicio de derechos dentro del espacio escolar, sea la institución que sea. Sin embargo, hay críticas por parte de los y las jóvenes y es que el derecho a la educación implica no sólo acceder sin restricciones, sino también aprender, saber y avanzar. Las acciones y la construcción de sentidos que los y las jóvenes de Caleta Olivia realizan dan cuenta del reclamo y la apuesta por ampliar estos derechos y demandar una formación más completa para el ejercicio de ciudadanía y a la vez atender las características y contenido de esa formación, así como también, los espacios de participación que se habilitan. Desde sus visiones, el desafío, en el contexto actual, implica apostar por la escuela de manera sostenida en términos de generación y multiplicación de espacios en donde se promueva el reconocimiento como sujetos de derecho y a sus prácticas culturales como parte constitutiva en la formación de su identidad ciudadana. La escuela, sin duda, estimula y garantiza ello en términos de participación en instancias de democratización. Por ello, en la medida en que pensemos la educación como un derecho y al Estado y a la 
escuela como quienes garantizan ese derecho no podemos pensarla si no es para todos y todas. $\mathrm{Y}$ en este sentido, observamos que los y las jóvenes sostienen ese sentido y dan peleas en su vida cotidiana para sostenerse en la escolaridad. Para ellos y ellas, la escuela es un espacio de encuentro, un lugar para estar a pesar de todo, un lugar que rescatan, al que quieren ir a pesar de todas las dificultades, un lugar en el que deciden estar y sostener.

En todo caso, el desafío desde sus visiones y en el contexto actual, implica apostar desde la escuela de manera sostenida a la generación y multiplicación de espacios en donde se promueva el reconocimiento como sujetos de derecho y a sus prácticas culturales como parte constitutiva en la formación de su identidad ciudadana, estimulando y garantizando la participación para ciudadanías que puedan no callarse, que puedan rebelarse, que puedan pugnar por entrar y ocupar lugares en un mundo en el que cada vez hay menos espacios para todos y todas.

\section{REFERENCIAS BIBLIOGRÁFICAS}

ABRATTE, J. (2015) "La educación como derecho: historia, política y desafíos actuales." Repositorio digital UNC. Recuperado de https://rdu.unc.edu.ar/handle/11086/5861/ discover?filtertype=subject\&filter_relational_operator=equals\&filter=Derecho+a+la+ Educación

APPADURAI, A. (2007). El rechazo de las minorías. Ensayo sobre la geografía de la furia. Ensayo TusQuets editores: Barcelona.

BARBERO, J. M. (2000). La ciudad entre medios y miedos. En S. Rotker (Ed), Ciudadanías del miedo (pp. 29-35). Caracas: Nueva Sociedad.

BAUDELOT, C. ESTABLET R. (1976) La escuela capitalista en Francia. Ed. S. XXI. Buenos Aires.

BAUMAN, Z. (2014). Miedo líquido. La sociedad contemporánea y sus temores. Buenos Aires Paidós.

BOWLES, S., \& GINTIS, H. (1981). La instrucción escolar en la América capitalista: la reforma educativa y las contradicciones de la vida económica.

BRASLAVSKY, C. (1989). Discriminación educativa en Argentina. Miño y Dávila, Buenos Aires, AR

BURGWALL, G. (1999). "Prácticas cotidianas de resistencia”. En Salman, T. y Kingman, E. (1999). Culturas urbanas e identidad. FLACSO. Ecuador

BUTLER, Judith. (2006). "Vida precaria: el poder del duelo y la violencia." Buenos Aires: Paidós.

BUTLER, Judith. (2017) "Cuerpos aliados y lucha política. Hacia una teoría performativa de la asamblea." Ed. Paidós Básica

CASTEL, R. (2014). Los riesgos de exclusión social en un contexto de incertidumbre. Revista internacional de sociología, 72(Extra_1), 15-24. https://doi.org/10.3989/ris.2013.03.18

CESTARE, M. (2007). "La experiencia escolar: ser alumno en emplazamientos urbano marginales". En Pedagogías desde América Latina: tensiones y debates contemporáneos. Ed. LEA: Bs. As.

CROCE, A. C. (2005). De las resistencias internas para incluir a los adolescentes y jóvenes en el sistema educativo. En M. Krichesky (Comp.), Adolescentes e inclusión educativa. Un derecho en cuestión (pp. 81-94). Buenos Aires: Ediciones Novedades Educativas

DAVIS, M. (2007). Planeta de ciudades miseria. Foca Ediciones: Madrid.

DE MARINIS P. (1999). "Gobierno, gubernamentalidad, Foucault y los anglofoucaultianos (o un ensayo sobre la racionalidad política del neoliberalismo)". 
DELEUZE, G. (1969) - Lógica del sentido Paidós, Barcelona/Buenos Aires

FOUCAULT, M. (1994). "La ética del cuidado de uno mismo como práctica de la libertad".En Hermenéutica del Sujeto. Ediciones La Piqueta: Madrid.

FOUCAUlT, M. (2006). Seguridad, Territorio. Población. Fondo de Cultura Económica. Bs. As.

GENTILI, P. (Abril 2009). Marchas y contramarchas. El derecho a la educación y las dinámicas de exclusión incluyente en América Latina (a sesenta años de la Declaración Universal de los Derechos Humanos). OEI- $\mathrm{N}^{\circ} 49$

GRINBERG, S. (2006). Educación y gubernamentalidad en las sociedades de gerenciamiento. Revista argentina de sociología, 4(6).

GRINBERG, S. (2008). "Educación y poder en el Siglo XXI. Gubernamentalidad y pedagogía en las sociedades de gerenciamiento" Ed. Miño y Dávila

GRINBERG S. (2009). "Políticas y territorios de escolarización en contextos de extrema pobreza urbana". En Rev. Archivos de Cs de la Educ., N³, UNLP, Pp 81-98.

KESSLER, G. (2002). La experiencia educativa fragmentada. Docentes y alumnos en la escuela media en Buenos Aires.

LANGER, CESTARE Y MARTINCIC (2017). Pobreza, educación, estigmatización y luchas por los derechos sociales. Trabajo presentado en $\mathrm{XIX}^{\circ}$ Congreso REDCOM "Federalizar la comunicación: experiencias, utopías y recorridos pendientes", Comodoro Rivadavia.

LANGER, E, ROLDÁN, S; MAZA, K. (2012) Dispositivos pedagógicos y trayectorias escolares en contexto de desigualdad social. ICT-UNPA-47-2012

LANGER, E; CESTARE, M; MARTINCIC, H. (2018). Ponencia "Luchas por el derecho a la educación en Caleta Olivia", presentadas en V Jornadas de investigación en Ciencias Sociales y humanidades UNSJB- Octubre 2018

LANGER, E. (2014). "Contraconductas y escolarización en contextos de pobreza urbana de Argentina”. En Revista Magistro. 7 (14). Pp. 297-334. Revista de la Maestría en Educación de la Vicerrectoría de Universidad Abierta y a Distancia - Universidad Santo Tomás. Bogotá D.C. ISSN: 2011-8643. Ediciones USTA. Disponible en http://revistas.usta.edu.co/index.php/magistro

LANGER, E. (2017). Escuela, pobreza y resistencia. Defensas y luchas cotidianas de estudiantes. Rada Tilly. Del Gato Gris. ISBN 978-987-46647-0-9.

LANGER, E. (2018). "Los miedos en tiempos de crisis según docentes y estudiantes de Argentina”. En Revista Universitas, 28, pp. 19-38. https://doi.org/10.17163/uni.n28.2018.01

LANGER, E., PÉREZ, A. Y CESTARE, M. (2015). Las luchas sociales por la escolarización en comunidades en condición de pobreza. Un estudio comparativo en Caleta Olivia (Santa Cruz) y José León Suárez (San Martín, Buenos Aires). En V Congreso Nacional e Internacional de Estudios Comparados en Educación. Educación y futuro. Debates y desafíos en perspectiva internacional. Organizado por la SAECE. 24 a 26 de Junio de 2015. https://doi.org/10.17163/uni.n24..2016.05

LANGER, E (2012). "Somos todos rebeldes. La ironía y el humor de estudiantes que resisten en contextos de pobreza urbana." Jornadas de jóvenes investigadores en educación FLACSO/Argentina.

LANGER, E. y CESTARE, M. (2015) Sostener la familia, luchar por la educación de sus hijos e insistir por tener lugar en la sociedad: relatos de mujeres en contextos de pobreza de la ciudad de Caleta Olivia/Santa Cruz.

LANGER, E; CESTARE, M; VILLAGRAN, C. (2015). "Estrategias y acciones en una escuela a la que concurren adolescentes y jóvenes de sectores populares en Caleta Olivia/Santa Cruz." 
LANGER, E; CESTARE, M. (2014). "Dispositivos pedagógicos, luchas de padres por la escolarización y contextos de pobreza urbana en Caleta Olivia / Santa Cruz." ALAS Volumen 6. Número 10.

LANGER, E. (2011). Nuevas aproximaciones teóricas a la noción de "resistencia" y sus Contribuciones al análisis de las regulaciones en los dispositivos pedagógicos del siglo XXI. In VIII Encuentro de Cátedras de Pedagogía de Universidades Nacionales Argentinas. Teoría, formación e intervención en Pedagogía.

LEMKE, T. (2006). "Marx sin comillas: Foucault, la gubernamentalidad y la crítica del neoliberalismo". En Lemke T., Legrand S., Le Blanc G., Montag, Giacomelli MMarx y Foucault. Claves perfiles. Ediciones Nueva Visión: Bs. As. Pp. 5-20.

LLOMOVATE, S., GUELMAN, A., LUCANGIOLI, A., KISILEVSKY, M., \& DUSCHATZKY, S. (1993). Escuela primaria y mundo del trabajo: Aportes, desde una sociología de la educación crítica en los 90.

MARGULIS, M., URRESTI, M. (1998). La construcción social de la condición de juventud. Viviendo a toda. Jóvenes, territorios culturales y nuevas sensibilidades, 3-21. Siglo del Hombre. Bogotá.

MALLIMACI, F., \& SALVIA, A. (Eds.). (2005). Los nuevos rostros de la marginalidad: la supervivencia de los desplazados. Editorial Biblos Sociedad.

O’MALLEY, P. (1996). Risk and responsibility. Foucault and political reason: Liberalism, neo-liberalism and rationalities of government, 189-207.

ONFRAY M. (2011). Política del rebelde. Tratado de resistencia e insumisión. Anagrama/ Colección Argumentos: Barcelona.

PEREA ACEVEDO, A. (2009). Estética de la existencia: Las prácticas de sí como ejercicio de la libertad, poder y resistencia en Michel Foucault.

PEREZ A. (2013) Configuraciones urbanas y escolares: notas de fragmentación educativa y territorial. La escuela Not Dead. Dispositivos pedagógicos, territorios y desigualdad. UNPA EDITA. RIO GALLEGOS

PINEAU, P., DUSSEL, I., \& CARUSO, M. (2001). La escuela como máquina de educar. Ed. Paidos. Buenos Aires.

PUIGGRÓS, A. (2003) El lugar del saber: conflictos y alternancias entre educación, conocimiento y política. Ed. Galerna. Bs As.

RINESI E., (2016) La educación como derecho. Notas sobre inclusión y calidad. En Inclusión y calidad como políticas educativas de Estado. O mérito como opción única de mercado. Brener G. y Galli G. (Comp.) Buenos Aires, Argentina. Ed Crujía

ROSE, N. (1996) ¿La muerte de lo social? Re-configuración del territorio de gobierno. Economy and society volume 25 number 3 .

ROTKER, S. (2000). Ciudadanías del miedo. Caracas: Editorial Nueva Sociedad.

SAINTOUT, F. (2009). Jóvenes, el futuro llegó hace rato: percepciones de un tiempo de cambios: familia, escuela, trabajo y política. Prometeo libros. Bs As.

SALAZAR PÉREZ, R. (2011). Los miedos ocultos en la sociedad del siglo XXI. Theomai, $23,24-34$

SCABUZZO, T. y FABRIZI, J. (2014). "El encuentro con la desigualdad: Teorías acerca de las causas." Recuperado de http://repositoriodigital.uns.edu.ar/bitstream/123456789/ 3815/1/Fabrizi\%2C\%20Scabuzzo.pdf

SENNETT, R. (2000). La corrosión del carácter. Las consecuencias personales del trabajo en el nuevo capitalismo. Barcelona, Anagrama.

TAYLOR, S. J. y BOGDAN, r. (1987) " introducción a los métodos cualitativos". Ed. Paidós 
TIRAMONTI, G. (2009). La escuela media en debate: problemas actuales y perspectivas desde la investigación / Guillermina Tiramonti y Nancy Montes. - 1a ed. - buenos Aires: manantial

TORRES, R. (2006). "Derecho a la educación es mucho más que acceso de niños y niñas a la escuela." Recuperado de https://www.google.com.ar/url?sa=t\&rct=j\&q=\&esrc= s\&source=web\&cd=1\&cad=rja\&uact=8\&ved=2ahUKEwiN0Nat0-LcAhUITZAKHR bOBY8QFjAAegQICBAC\&url=https\%3A\%2F\%2Fwww.oei.es\%2Fhistorico\%2Finic ial\%2Farticulos\%2Fderecho

USECHE ALDANA, O. (2008). Miedo, seguridad y resistencias: el miedo como articulación Política de la negatividad. Polis, 19. Recuperado de https://goo.gl/2bSxT7 (27-092017)

VENTURINI, M. E. (2007). "Configuraciones barriales-escolares en la era del gerenciamiento en contextos de pobreza”. En Pedagogías desde América Latina: tensiones y debates contemporáneos. Ed. LEA: Bs. As.

VILLAGRAN, C. (2016) Curriculum y regulación escolar: los procesos de reforma y los Docentes. Un estudio en la escuela primaria y secundaria en la ciudad de Caleta Olivia. (Tesis de Maestría) UNPA. 